\title{
Fractioned Stereotactic Radiosurgery in Patients with Paraganglioma
}

\section{Paragangliomalı Hastalarda Fraksiyone Stereotaktik Radyoterapi}

\author{
Ayşen Dizman, Ebru Atasever Akkaş, Gökçe Kaan Olcay, Emine Keven \\ Ankara Dr. Abdurrahman Yurtarslan Onkoloji Eğitim ve Araştırma Hastanesi, Radyasyon Onkolojisi Kliniği, \\ Ankara \\ Dergiye Ulaşma Tarihi: 02.06.2016 Dergiye Kabul Tarihi: 04.11.2016 Doi: 10.5505/aot.2017.29292
}

\begin{abstract}
ÖZET
GİRIŞ ve AMAÇ: Paraganglioma, nadir görülen, ağrısız, yoğun vasküler yapıya sahip tümördür. Paraganglioma için tedavi seçenekleri, cerrahi, konvansiyonel radyoterapi, stereotaktik radyocerrahi ya da bu modalitelerin kombinasyonudur. $\mathrm{Bu}$ çalışmanın amacı, farksiyone stereotaktik radyoterapi ile tedavi edilen paragangliomalı hastaların tedavi sonuçlarını değerlendirmektir.

YÖNTEM ve GEREÇLER: Kliniğimizde tedavi edilen, fraksiyone stereotaktik radyoterapi yöntemi ile tedavi edilen dokuz hasta değerlendirildi. İki hasta kadın, diğerleri erkekdi. Uygulanan tedavi dozu median 5 fraksiyonda 25 Gy idi.

BULGULAR: Median takip 65 ay (46-71 ay) di. Hastaların tümü tedavi sonras 6 ayda bir manyetik rezonans görüntüleme(MRI) ile değerlendirildi. Lezyonlar 3 hastada stabil iken, 1 hastada tam cevap, diğer beş hastada parsiyel cevap elde edildi. Hastaların hiçbirinde akut toksisite görülmedi. İki hastada ise takiplerde geç toksisite görüldü.

TARTIŞMA ve SONUÇ: Bizim deneyimlerimize göre, paraganglioma tedavisinde, CyberKnife ${ }^{\circledR}$ (Accuray Incorporated, Sunnyvale, CA) ile fraksiyone stereotaktik radyoterapinin, başarılı bir tedavi seçeneği olduğu görülmüştür.
\end{abstract}

Anahtar Kelimeler: paraganglioma, stereotactic radyocerrahi, radyoterapi

ABSTRACT
INTRODUCTION: Paraganglioma are rare, indolent and highly vascular tumors. The treatment options for paraganglioma include surgery, conventional external beam radiotherapy (EBRT), stereotactic radio-surgery (SRS) or combination of these modalities. EBRT has been used to treat unresectable tumors and patients with residual or recurrent lesions after surgery The aim of this study is evaluated the results of treatment in patients with paraganglioma who were treated with fractioned stereotactic radiotherapy.

METHODS: Nine patients with paraganglioma, who were treated with fractioned stereotactic radiotherapy, were retrospectively evaluated.Two patient was male, and others were female. The median dose to the tumor was 25 Gy in median 5 fractions.

RESULTS: Median follow-up was 65 months (range, 46-71 months). All patients were evaluated with magnetic rezonance imaging, every 6 months. Lesions were stable in 3 patients, and it was observed complete response in 1 patient and partial response in 5 patients. We did not observe any treatment related acute toxicity in our patients. We observed late toxicity in 2 patients.

DISCUSSION AND CONCLUSION: In conclusion, according to our experience, fractioned stereotactic radiotherapy with CyberKnife ${ }^{\circledR}$ (AccurayIncorporated, Sunnyvale, CA) seems to be successful treatment option in the management of paraganglioma.

Keywords: paraganglioma, stereotactic radiosurgery, radiotherapy

\section{Giriş:}

Paraganglioma, nadir görülen, ağrısız ve yoğun vasküler yapıya sahip tümördür. Kemodektoma ya da glomus tümörü olarak da bilinir. Kromafin hücrelerinin bulunmasina sekonder olarak katekolaminleri salgilayabilirler ve buna bağlı olarak labil hipertansiyon ve taşikardi gibi semptomlara neden olabilirler (1). Baş boyun bölgesinde görülen tüm baş boyun tümörlerinin $\% 0,06$ sin1 oluşturur. Genellikle 3. ve 6. dekat arasında görülür. Olguların çoğu benign olmakla birlikte, literatürde $\% 10$ dan az oranda malign olarak da bildirilmiştir. Metastaz en 
sıklıkla servikal lenf nodlarında nadiren de akciğer, karaciğer, kemik, cilt gibi uzak bölgelerde görülebilir (2).

Baş boyun bölgesinde yerleşen paragangliomaların klinik belirtileri, lokalizasyonuna bağlı olarak değişebilir. Karotid gövde tümörleri, karotid arterin bifurkasyonuna doğru büyüyen ve genellikle boyunda yavaş büyüyen pulsatil kitle olarak ortaya çıkar. Baş boyun paragangliomalarının büyük bir kısmını bu lokalizasyondaki tümörler oluşturur.Temporal kemik paragangliomu ya da glomus timpanikum tümörleri genellikle orta kulak boyunca uzanan timpanik (Jacobsen's) veya aurikuler (Arnold's) sinirlerden köken alır. Genellikle işitme kaybı, tinnitus, denge kaybına neden olabilir ve fizik muayenede kulak zarı arkasında kitle olarak görülebilir. Glomus jugulare (GJ) tümörleri ise kafa tabanında, jugular bulb den köken alır ve kemik destrüksiyonu ile birlikte olabilir. Bu tümöre sahip olan hastalarda genellikle 9. ve 12 . kraniyal sinir defisiti görülebilir. GJ tümörleri ayrıca timpanik kanaldan orjin alabilir ya da bu bölgeye yayılabilirler (3).

Baş boyun paragangliomalarının güncel tedavi şekli, endovasküler embolizasyon aracılığ 1 ile cerrahi, konvansiyonel radyoterapi, stereotaktik radyocerrahi ya da bu modalitelerin kombinasyonudur (4). Cerrahide amaç, tümörün komplet çıkarılmasıdır. Buna rağmen nüks oranları \%3-20 arasında değişmektedir (57).

Fraksiyone eksternal radyoterapi daha çok nüks ya da rezüdü tümör varlığında, bazen de opere edilemeyen hastalarda primer tedavi modalitesi olarak kullanılmaktadır (8). Tümör kontrol oranları \%74-97 arasında değişmektedir (8-11). Definitif RT de kür tanımı, tümörün progrese olmasını engellemektir. Tümör çok yavaş regrese olabilir ancak tamamen kaybolmayabilir (3).

Son iki dekat boyunca bu tümörlerin tedavisinde stereotaktik radyocerrahi (SRS) ya da stereotaktik fraksiyone radyoterapi (SFRT) kullanılmaya başlamıştır (12). Radyocerrahi, yüksek derecede güvenli ve hassas bir tedavi yöntemidir. Hedef volüm çevresinde hızlı doz düşüşü özelliği nedeniyle tümöre yüksek doz verebilmeye izin verirken, çevre sağlam dokuda minimum hasar oluşturmaktadır. $\mathrm{Bu}$ da özellikle paraganglioma gibi benign tümörlerin tedavisinde önem taşımaktadır (13).

\section{Gereç ve Yöntemler:}

Haziran 2009-Mayıs 2012 tarihleri arasinda kliniğimizde tedavi edilen 9 hasta çalışmaya dahil edildi. Tüm hastalara radyolojik olarak tanı konmuştu. Hastaların 2 si kadın, diğerleri erkekdi. Ortanca yaş 54 (30-62) idi. Bir hasta daha önce opere olmuştu. Üç hastaya ise daha önce konvansiyonel RT uygulanmıştı. Diğer beş hasta ise ilk kez tedavi edildi. Hastalara ait karakteristik özellikler Tablo 1 de gösterilmiştir (Tablo 1).

Hastaların tümüne CyberKnife ${ }^{\circledR}$ (Accuray Incorporated, Sunnyvale, CA) ile fraksiyone stereotaktik RT (FSRT) uyguland. İlk olarak tüm hastalara immobilizasyon amaçlı termoplastik baş- boyun maskesi yapılarak ince kesit $(1,25 \mathrm{~mm})$ bilgisayarlı tomografi (BT) çekimi yapıldı. Daha sonra, aynı pozisyonda (aynı maske kullanılarak) kontrastlı T1 ağırlıklı manyetik rözenans görüntüleme (MRI) leri alındı. Görüntüler CyberKnife ${ }^{\circledR}$ tedavi planlama istasyonuna aktarılarak füzyon yapıld1. Bu görüntüler üzerinde hedef volüm ve çevre kritik organlar konturland.

Tedavi planları, MultiPlan 2.1.0 tedavi planlama sisteminde ray-tracing doz hesaplama algoritması kullanılarak yapıldı (Resim 1,2).

Tedavi, altı hastaya 3 fraksiyonda, üç hastaya ise 5 fraksiyonda ortanca 24 Gy (18-29 Gy) gün aşırı olacak şekilde uygulandı. Reçete edilen izodoz \%83-92 arasinda, kapsama \%92-100, konformite indeks 1.47-2.3 arasinda değişmekte idi (Tablo 2).

\section{Bulgular:}

Tedavi sonrası hastaların takibi 6 ayda bir MRI ile yapildı. Ortanca takip süresi 65 ay (46-71 ay) idi. Bir hastada tam yanit, 5 hastada parsiyel yanıt elde edilirken, 3 hastada hastalık stabil kald1. Parsiyel yanit elde edilen bir hastada 4 . yıldan sonra tümörde büyüme saptandı ve başka bir merkezde opere edildi. Tedavi boyunca ve takip eden 3 ay lik dönemde hiçbir hastada akut komplikasyon gözlenmedi. Son kontrol muayenelerinde bir hasta dişında progresyon saptanmadi. Lokal kontrol oran1 \%89 olarak belirlendi. 2. seri RT uygulanan bir hastada tedaviyi takiben 3.y1lda 9. ve 12. kraniyal sinir toksisitesine bağlı yutma güçlüğü ve beslenmesi bozukluğu görüldü. Bir hastamızda ise tedaviden sonraki 8. ayda kalıcı fasiyal paralizi 
gelişti. Tedavi parametreleri Tablo 2' de yer almaktadır.

Tablo 1: Hastalara ait klinik ve demografik özellikler

\begin{tabular}{|c|c|c|c|c|}
\hline Hasta No & Yaş & Cinsiyet & Tümör Volümü (cm $\left.{ }^{\mathbf{3}}\right)$ & Tedavi Şekli \\
\hline 1 & 58 & $\mathrm{~K}$ & 14.240 & İk tedavi \\
\hline 2 & 54 & $\mathrm{~K}$ & 1.009 & İlk tedavi \\
\hline 3 & 49 & $\mathrm{~K}$ & 2.524 & İlk tedavi \\
\hline 4 & 38 & $\mathrm{~K}$ & 15.087 & Post op \\
\hline 5 & 35 & $\mathrm{E}$ & 3.453 & İk tedavi \\
\hline 6 & 30 & $\mathrm{~K}$ & 24.583 & 2. Seri \\
\hline 7 & 61 & $\mathrm{~K}$ & 28.535 & İlk tedavi \\
\hline 8 & 62 & $\mathrm{E}$ & 12.714 & 2 . Seri \\
\hline 9 & 62 & $\mathrm{~K}$ & 56.844 & 2. Seri \\
\hline
\end{tabular}

Tablo 2: Tedavi özellikleri

\begin{tabular}{|c|c|c|c|c|}
\hline Hasta No & $\begin{array}{c}\text { Tedavi } \\
\text { Dozu(Gy) }\end{array}$ & $\begin{array}{c}\text { Fraksiyon } \\
\text { Sayısı }\end{array}$ & $\begin{array}{c}\text { Reçete edilen } \\
\text { izodoz(\%) }\end{array}$ & CI \\
\hline 1 & $26 \mathrm{GY}$ & 3 & 91 & 1,6 \\
\hline 2 & $25 \mathrm{GY}$ & 5 & 92 & 1,47 \\
\hline 3 & $24 \mathrm{GY}$ & 3 & 87 & 1,6 \\
\hline 4 & $18 \mathrm{GY}$ & 3 & 80 & 2,1 \\
\hline 5 & $24 \mathrm{GY}$ & 3 & 85 & 1,65 \\
\hline 6 & $29 \mathrm{GY}$ & 5 & 83 & 1,95 \\
\hline 7 & $24 \mathrm{GY}$ & 3 & 93 & 2,3 \\
\hline 8 & $24 \mathrm{GY}$ & 3 & 90 & 1,7 \\
\hline 9 & $25 \mathrm{GY}$ & 5 & 85 & 2,1 \\
\hline
\end{tabular}

CI: Conformity index

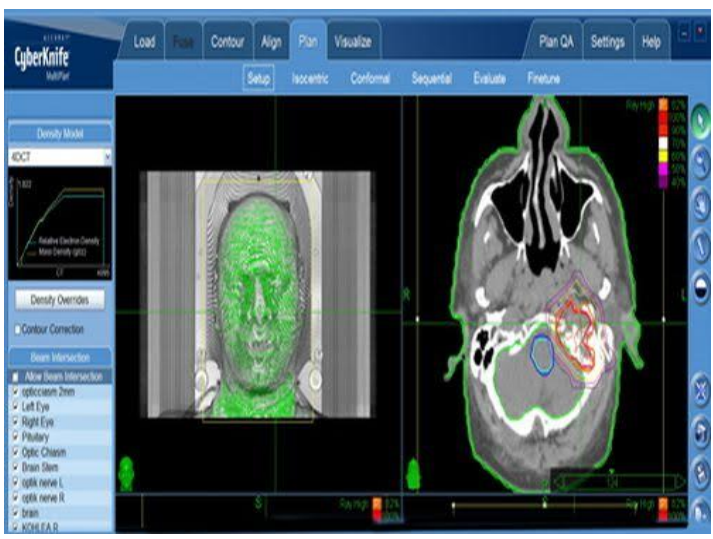

Resim 1: Tedavi planlama görüntüsü

\section{Tartışma:}

Baş boyun paragangliomalarının güncel tedavi şekli, endovasküler embolizasyon aracılığı ile cerrahi, konvansiyonel radyoterapi, stereotaktik radyocerrahi ya da bu modalitelerin kombinasyonudur (9). Cerrahi tekniklerde meydana gelen gelişmelere rağmen, mortalite

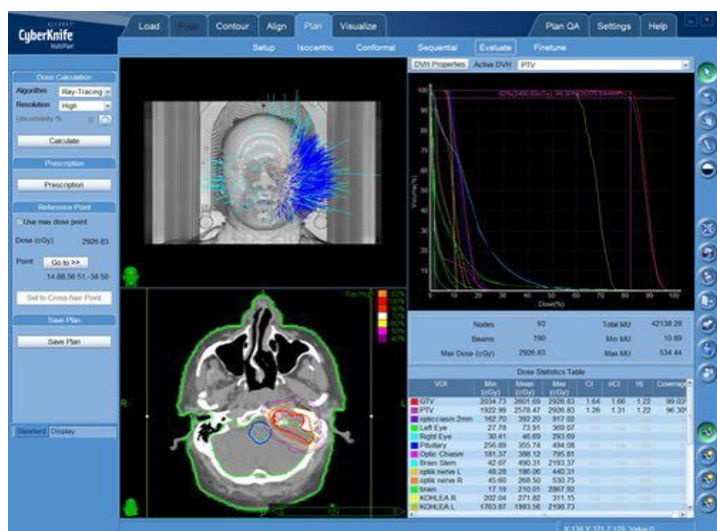

Resim 2: Tedavi planlama, kritik organ dozları ve doz volüm histogramı

oranı halen yüksek seyretmektedir (14-17). Özellikle, postoperatif IX. ve XII. kraniyal sinir hasar1 \%31-81 oranında bildirilmektedir. Ayrıca \%16-23 oranında fasiyal sinir paralizisi, \%6 oranında VI. sinir hasarı, \%4-26 oranında işitme kayb1 bildirilmiştir (14-23). Bu tümörlerdeki yüksek vaskülarizasyon nedeniyle rezeksiyon sirasinda tehlikeli boyutlarda 
kanama olabilir (22). Cerrahi mortalite oranları \%4-6,4 arasinda bildirilmektedir (17,23-25). Bu nedenle cerrahi tedavi teknik olarak güçtür ve önemli riskler taşımaktadır.

Paraganglioma tedavisinde kullanılan bir diğer tedavi modalitesi radyoterapidir. Cerrahi sonrası ya da tek başına uygulanabilmektedir. Son dönemlerde Suarez ve arkadaşları tarafından yayınlanan bir derlemede, juguler ve vagal paragangliomaların tedavisinde; RT ile, cerrahiye göre lokal kontrol oranlarının daha yüksek $(\mathrm{p}=.002)$ ve morbiditenin daha düşük olduğu $(\mathrm{p}=.003)$ belirtilmiştir (26).

Radyoterapi tekniklerindeki geniş spektrum, bu tedavi yöntemi için farkl1 alternatifleri sunabilmektedir. Son dönemlerde, konvansiyonel radyoterapi teknikleri dışında, stereotaktik radyoterapi teknikleri de kullanılmaya başlanmıştır. Paraganglioma gibi benign tümörlerin radyoterapisinde, hedef volüm, sadece tümör dokusudur ve geniş tedavi alanlarının tedavi edilmesine gerek yoktur. Stereotaktik radyocerrahi (SRS) ya da fraksiyone stereotaktik radyoterapi(FSRT) gibi teknikler ile, radyasyon, tanımlanmış hedef volüme güvenli bir biçimde uygulanırken, diğer taraftan hedef volüm dışında hızlı doz düşüşü nedeniyle komşu sağlam dokular daha iyi korunabilmektedir. Stereotaktik tedavi; Gamaknife, Cyberknife, ya da Linac bazlı cihazlar ile yapilabilmektedir.

Paragangliomalarda (glomus tümörü) GamaKnife ile stereotaktik radyoterapi sonuçları ilk olarak 1995 'de yayınlanmıştır (27). Daha sonra da çeşitli çalışmalar ile cesaret verici sonuçlar literatürde yer almaya başlamıştır (28-36). Gerosa ve ark (37); Gama Knife ile lokal kontrol oranları ve klinik cevabın, fraksiyone radyoterapi ile elde edilen sonuçlara benzer hatta daha iyi olduğunu belirtmiştir. Aynı zamanda, bu uygulama ile radyasyona maruz kalan normal doku hacminin daha küçük olması nedeniyle kronik komplikasyon oranlarının daha az olduğu rapor edilmiştir.

Bir derlemede, Gama Knife ile tedavi edilen, beş ayrı merkezden 133 hastanın sonuçları değerlendirilmiştir. Ortalama 31 ay (24-50 ay)'l1k takipte, \%100 lokal kontrol, $\% 23-50$ regresyon ve \%38-77 de stabil hastalık oranları elde edilmiştir. Aynı zamanda hastaların \%96'sında nörolojik iyileşme olduğu belirtilmiştir (38).

İbrahim R ve arkadaşları tarafından yapılan ve 2016 yılında yayınlanan bir çalışmada(39), Gama Knife ile stereotaktik radyocerrahi uygulanan 75 olgunun sonuçları, retrospektif olarak değerlendirilmiştir. Ortanca doz, tek fraksiyonda 18 Gy (12-25 Gy), ortanca radyolojik takip süresi 51.5 ay (12-230 ay) olarak belirtilmiştir. Ortalama tumor hacmi 9.1 $\mathrm{cm}^{3}$ dür. En son takip MRI sonuçlarına göre, tumor kontrol oran1 \%93.4 (43 hastada, tümör boyutunda azalma; 28 hastada, stabil hastalı; 5 hastada tümör boyutunda artış) iken 2,5 ve 10 yıllık progresyonsuz sağkalım oranları sırasıyla $97.2 \%, 92.2 \%$ ve $86.3 \%$ 'dır. Tedaviye bağlı iki hastada kalıc1 kraniyal sinir hasarı(bir hastada vokal kord paralizisi, diğerinde fasiyal sinir paralizisi) gelişmiştir. 13 hastada ise, tedaviyi takiben 5-8 ay lik dönemde ortaya çıkan ve 1-2 haftalık steroid tedavisi ile kaybolan geçici kraniyal sinir hasarı (V, VII, X) rapor edilmiştir. Bu çalışmada, $>7 \mathrm{~cm}^{3}$ tümör hacminin, kraniyal sinir hasarı açısından risk artışına neden olduğu gösterilmiştir ( $\mathrm{p}=0.038$ ).

Livia Binachi ve ark; tarafindan 2009 da cyberknife ile tedavi edilen 9 glomus jugulare tümörlü hastanın tedavi sonuçları yayınlanmıştır. 8 hastaya, tek fraksiyonda ortalama 12.5 Gy (11-13 Gy), bir hastaya ise 3 fraksiyonda toplam 24 Gy stereotaktik tedavi uygulanmıştır. Ortalama tümör volümü $6,7 \mathrm{~cm}^{3}$ dür. Ortalama 20 aylık takip boyunca 8 hastada(bir hasta farkl1 bir nedenle kaybedilmiş) lokal kontrol sağlandığ1 belirtilmiştir. Hiç bir hastada ek bir nörolojik defisit gelişmemiştir. İki hastada tedavi sonras1 geçici başağrısı meydana gelmiştir (40).

Lim $M$ ve arkadaşlarının çalışmasında, Cyberknife ya da LINAC (Linear Accelerator) ile stereotaktik radyoterapi uygulanan 21 glomus jugulare tümörünün sonuçları yayınlanmıştır. Ortalama takip süresinin 60 ay olduğu çalışmada, tedavi dozu 14 Gy ile $27 \mathrm{~Gy}$ arasında değişmektedir. Tümör, olguların tümünde stabil kalmış ya da boyutları azalmıştır. Bir hastada geçici olarak dilde güçsüzlük ve işitme kaybı görüldüğü belirtilmiştir (41).

Cyberknife, stereotaktik radyocerrahi/ radyoterapi uygulamasına olanak sağlayan bir sistemdir. İlk olarak 2004'de kullanılmaya başlanmıştır. Gama Knife'a göre bazı 
avantajlara sahiptir. Gama Knife'da; tedavi öncesi invaziv bir işlemle yerleştirilen ve "frame" adı verilen çerçeve takılmaktadır. İşlemin invaziv olması nedeniyle, tedavi yüksek dozda tek fraksiyonda uygulanmaktadır. CyberKnife uygulamalarında ise bu şekilde bir çerçeve yerleştirilmeden, baş, termoplastik bir maske ile immobilize edilmektedir. $\mathrm{Bu}$ da tedavinin tekrarlanabilirliğine olanak sağlamaktadır. Böylece tedavi çoklu fraksiyonda daha düşük fraksiyon dozları ile yapılmakta ve geç yan etkiler azalmaktadır.

Biz de çalışmamızda, CyberKnife (Accuray, Inc., Sunnyvale, CA) ile fraksiyone stereotaktik radyoterapi uyguladığımız paraganglioma tanılı dokuz hastamızın sonuçlarını retrospektif olarak değerlendirdik. Son değerlendirmede sekiz hastada tümör kontrolü devam ediyordu. Bir hastada ise tedavi sonras1 dördüncü yılda progresyon gelişti. Lokal kontrol oran1 \%89 olarak belirlendi. Takiplerde iki hastada kraniyal sinir defisiti gelişti(\%22.2). Bunlardan birinde tümör hacmi diğerlerinin yaklaşık iki katı idi ve dolayısıyla daha geniş bir alan tedavi edilmek zorunda kalındığından çevredeki normal dokular yeterince korunamamıştı. Diğerinde ise ikinci seri tedavi uygulanmıştı. Tedavi sonuçları, literatür ile oransal olarak karşılaştırıldığında benzer lokal kontrol oranları sağlandığ 1 görülmektedir. Ancak toksiste oranlarımız

\section{Kaynaklar}

1. Baysal BE. Hereditary paraganglioma targets diverse paraganglia. J Med Genet. 2002; 39: 617-622

2. Lee JH, Barich F, Karnell LH, Robinson RA, Zhen WK, Gantz BJ, Hoffman HT. American College of Surgeons Commission on Cancer, American Cancer Society National Cancer Data Base report on malignant paragangliomas of the head and neck. Cancer. 2002; 94:730-737

3. Philip Gilbo, Christopher G. Morris, Robert J, et al. Amdur. Radiotherapy for Benign Head and Neck Paragangliomas:A 45-Year Experience. Cancer. 2014; 1: 3738-3743

4. Chapman DB, Lippert D, Geer CP, et al. Clinical, histopathologic,and radiographic indicators of malignancy in head and neck paragangliomas.Otolaryngol Head Neck Surg. 2010;143:531-537.

5. Springate SC, Weichselbaum RR. Radiation or surgery for chemodectoma of the temporal bone: a review of local control and complications. Head Neck. 1990; 12(4): 303-307 literatürde belirtilen oranlardan daha yüksek olduğu görülmektedir. $\mathrm{Bu}$ durumun toksisite gelişen olgulardan birinin tümör hacminin çok büyük olmasına $\left(56.844 \mathrm{~cm}^{3}\right)$, diğerinde ise ikinci seri tedavi olmasına bağlıyoruz. $\mathrm{Bu}$ da literatürde de belirtildiği gibi büyük tümör hacminin ve ikinci seri tedavinin toksisite oranlarını arttırdığı bilgisini desteklemektedir.

Çalışmamızın, az sayıda vaka içeriyor olması, retrospektif değerlendirme olması ve kısa süreli takip gibi limitasyonları bulunmaktadır. Ancak; literatürde paraganglioma tedavisinde Cyberknife ile uygulanan stereotaktik radyoterapi sonuçlarının az sayıda olması nedeniyle, bu alanda katk1 sağlayabileceğini düşünmekteyiz.

\section{Sonuçlar}

Sonuç olarak, bizim sonuçlarımıza ve literatür sonuçlarına dayanarak paraganglioma tedavisinde cyberknife ile stereotaktik radyoterapi uygulamasının iyi bir alternatif tedavi yöntemi olduğunu söyleyebiliriz.

\section{Çıkar Çatışması: Yok}

6. Gottfried ON, Liu JK, Couldwell WT. Comparison of radiosurgery and conventional surgery for the treatment of glomusjugulare tumors. Neurosurg Focus. 2004; 15:17(2):E4

7. Chretien PB, Engelman K, Hoye RC, Geelhoed GW Surgical management of intravascular glomus jugulare tumor. Am J Surg. 1971; 122:740-743

8. Boyle JO, Shimm DS, Coulthard SW. Radiation therapy for paragangliomas of the temporal bone. Laryngoscope. 1990;100:896-901.

9. Raygada M, Pasini B, Stratakis CA. Hereditary paragangliomas. Adv Otorhinolaryngol. 2011;70: 99-106.

10. Cole JM, Beiler D. Long-term results of treatment for glomus jugulare and glomus vagale tumors with radiotherapy. Laryngoscope. 1994;104:1461-1465.

11. Cummings BJ, Beale FA, Garrett PG, et al. The treatment of glomus tumors in the temporal bone by megavoltage radiation. Cancer. 1984;53:2635-2640.

12. Henzel M, Hamm $K$, Gross MW, et al. Fractionated stereotactic radiotherapy of glomus jugulare tumors: Local control, toxicity, symptomatology, and quality of life. Strahlenther Onkol. 2007; 183:557-562. 
13. Zachary D. Guss, A.B., Sachin Batra, M.B.B.S., Charles J. Limb, et al. Radiosurgery of glomus jugulare tumors: A Metaanalysis. Int J Radiat Oncol Biol Phys. 2011; 15; 81(4): e497-e502

14. Cece JA, Lawson W, Biller HF, Eden AR, Parisier SC. Complications in the management of large glomus jugulare tumors. Laryngoscope. 1987; 97(2):152-157

15. Gjuric $M$, Rüdiger Wolf $S$, Wigand $M E$, Weidenbecher M. Cranial nerve and hearing function after combined-approach surgery for glomus jugulare tumors. Ann Otol Rhinol Laryngol. 1996;105(12):949-954

16. Jackson CG, McGrew BM, Forest JA, Netterville JL, Hampf CF, Glasscock ME Lateral skull base surgery for glomus tumors: long-term control. Otol Neurotol. 2001; 22:377382

17. Watkins LD, Mendoza N, Cheesman AD, Symon L. Glomus jugulare tumours: a review of 61 cases. Acta Neurochir(Wien). 1994; 130:6670

18. Al-Mefty O, Teixeira A. Complex tumors of the glomus jugulare: criteria, treatment, and outcome. J Neurosurg. 2002 97:1356-1366 Pfeiffer J. Neuropathologie, 1984; vol 4. Springer, Heidelberg

19. Thabet $\mathrm{MH}$, Kotob H. Cervical paragangliomas: diagnosis, management and complications. $\mathrm{J}$ Laryngol Otol 2001;115:467-474

20. de Jong AL, Coker NJ, Jenkins HA, Goepfert H, Alford BR et al. Radiation therapy in the management of paragangliomas of the temporal bone. Am J Otol.1995; 16:283-289

21. Springate SC, Haraf D, Weichselbaum RR. Temporal bone chemodectomas-comparing surgery and radiation therapy. Oncology (Williston Park) 1991; 5:131-137

22. Young NM, Wiet RJ, Russell EJ, Monsell EM. Superselective embolization of glomus jugulare tumors. Ann Otol Rhinol. Laryngol. 1988; 97:613-620

23. Gjuric M, Seidinger L, Wigand ME. Long-term results of surgery for temporal bone paraganglioma. Skull Base Surg. 1996; 6:147152

24. Green JD Jr, Brackmann DE, Nguyen CD, Arriaga MA, Telischi FF, De la Cruz A et al. Surgical management of previously untreated glomus jugulare tumors. Laryngoscope. 1994; 104:917-921

25. Raquet F, Mann W, Maurer J, Gislbach J Functional deficits of caudal cranial nerves after surgery of tumors in the foramen jugulare. A long-term follow-up study. Laryngorhinootologie. 1991; 70:284-288

26. Suarez C, Rodrigo JP, Bodeker CC, et al. Jugular and vagal paragangliomas:systematic study of management with surgery and radiotherapy. Head Neck. 2013;35:1195-1204.

27. Kida, Y., Kobayashi, T., Tanaka, T., Oyama, H., Niwa, M. A new strategy for the treatment of jugular foramen tumors using radiosurgery. No Shinkei Geka. 1995; 23: 671-675
28. Jordan J. A., Roland P. S., McManus C., Weiner R. L., Giller C. A. Stereotastic radiosurgery for glomus jugulare tumors. Laryngoscope. 2000; $110: 35-38$

29. Mukherji S. K., Kasper M. E., Tart R. P., Mancuso A. A. Irradiated paragangliomas of the head and neck: CT and MR appearance. AJNR Am J Neuroradiol. 1994;15: 357-363

30. Eustacchio S., Leber K., Trummer M., Unger F., Pendl G. Gamma knife radiosurgery for glomus jugulare tumours. Acta Neurochir (Wien). 1999; 141: 811-818

31. Eustacchio S., Trummer M., Unger F., Schrottner O., Sutter, B.,Pendl, G. The role of Gamma Knife radiosurgery in the management of glomus jugular tumours. Acta Neurochir Suppl. 2002; 84: 91-97

32. Feigenberg S. J, Mendenhall Hinerman R, et al. Radiosurgery for paraganglioma of the temporal bone. Head Neck 2002; 24:384-389

33. Foote R. L, Coffey R. J, Gorman D.A, et al. Stereotactic radiosurgery for glomus jugulare tumors: a preliminary report. Int J Radiat Oncol Biol Phys. 1997; 38: 491-495

34. Foote R. L, Pollock B.E, Gorman D.A et al. Schomberg, P. J., Stafford, S. L., Link, M. J., Kline R. W., Strome S. E., Kasperbauer J.L., Olsen K. D. Glomus jugulare tumor: tumor control and complications after stereotactic radiosurgery. Head Neck. 2002; 24: 332-338; discussion 338-339

35. Liscak R, Vladyka V., Simonova G., Vymazal J., Janouskova L. Leksell gamma knife radiosurgery of the tumor glomus jugulare and tympanicum. Stereotact Funct Neurosurg.1998; 70: Suppl 1, 152-160

36. Liscak R., Vladyka V., Wowra B., et al. Gamma Knife radiosurgery of the glomus jugulare tumour - early multicentre experience. Acta Neurochir(Wien). 1999; 141: 1141-1146

37. Gerosa M, Visca A, Rizzo P, Foroni R, Nicolato A, Bricolo A. Glomus jugulare tumors: the option of gamma knife radiosurgery. Neurosurgery. 2006; 59:561-569 (discussion 561-569)

38. Sheehan J, Kondziolka D, Flickinger J, Lunsford LD. Gamma knife surgery for glomus jugulare tumors: an intermediate report on efficacy and safety. J Neurosurg. 2005; 102(Suppl):241-246 484 Neurol Sci 2009; 30:479-485

39. Livia C. B., Marcello M., Lorenzo B., et al. Paragangliomas of head and neck: a treatment option with CyberKnife radiosurgery. Neurol Sci.2009; 30:479-485.

40. Ramez I, Mohannad B.A, John Y et al. Gamma Knife radiosurgery for glomus jugulare tumors:a single-center series of 75 cases. J Neurosurg. 2016; 8:1-10.

41. Lim M, Bower R, Nangiana JS et al. Radiosurgery for glomus jugulare tumors. Technol Cancer Res Treat. 2007 Oct;6(5):419-23. 